\title{
TOPOLOGICAL FROBENIUS PROPERTIES FOR NILPOTENT GROUPS. II
}

\author{
EBERHARD KANIUTH
}

\section{Introduction.}

Much work has been done on generalizing the classical Frobenius reciprocity theorem to non-compact groups. Given a locally compact group $G$, one can contemplate the following topological Frobenius property (FP): For every closed subgroup $H$ of $G$ and each pair of irreducible unitary representations $\pi$ and $\tau$ of $G$ and $H$, respectively, $\pi$ is weakly contained in the induced representation $\operatorname{ind}_{H}^{G} \tau$ if and only if the restriction $\pi \mid H$ weakly contains $\tau$ [10]. This is far from holding in general. Indeed, (FP) to hold for $G$ amounts to $G$ being a group with relatively compact conjugacy classes $[8,14]$. However, for many representations $\pi$ or for special classes of locally compact groups (FP) or at least part of it may still be valid.

To fix terminology let us say that an irreducible representation $\pi$ of $G$ satisfies (FP1)(resp. (FP2)) provided that the if (resp. only if) direction of (FP) holds (for all $H, \tau$ as above). For connected groups it is also of interest to consider the versions (FPC1) and (FPC2) of (FP1) and (FP2), repectively, that are obtained by restricting $H$ to connected subgroups of $G$. (FP1) has been investigated for discrete groups [14], for motion groups [15], and for nilpotent groups [2]. This paper deals with the more intricate property (FP2) for nilpotent groups and can be regarded as a counterpart to [2].

A brief outline of the paper is as follows. Let $G_{F}$ denote the subgroup of $G$ consisting of all elements with relatively compact conjugacy classes, and let $\pi$ be an irreducible representation of $G$. We first notice that if $\pi$ is weakly equivalent to $\operatorname{ind}_{N}^{G}(\pi \mid N)$ for some closed normal subgroup $N$ of $G$ contained in $G_{F}$, then (FP2) holds for $\pi$. The converse turns out to be true if either $G$ is a finitely generated nilpotent discrete group (Theorem 1.2) or if $G$ is connected nilpotent and $\pi$ is square integrable modulo its kernel (Theorem 2.5). In Section 3 we study nilpotent groups of the form $R \ltimes \mathrm{R}^{d}$ and establish necessary and sufficient conditions for $\pi$ to satisfy (FP2)(Theorem 3.4). This result shows that (FP2) is not

Received March 3, 1990. 
as rare a property as expected and seems to indicate that for a simply connected nilpotent group and a fixed $\pi$, FPC2) implies (FPC1). That this is not the case is demonstrated in Section 4 by looking at the 3-step nilpotent group of upper triangular matrices.

\section{Preliminaries and Nilpotent Discrete Groups.}

Let $G$ be a locally compact group. Our notation for the ascending central series of $G$ will be standard: $Z(G)=Z_{1}(G) \subset Z_{2}(G) \subset \ldots$ Also, for $A, B \subset G,[A, B]$ is the set of all commutators $[x, y], x \in A, y \in B$. In what follows the (not necessarily closed) normal subgroup $G_{F}$ of $G$ consisting of all elements with relatively compact conjugacy classes will play an important role.

We will use the same letter, say $\pi$, to denote a unitary representation of $G$ and the corresponding *-representation of the group $C^{*}$-algebra $C^{*}(G)$, and ker $\pi$ means the kernel of $\pi$ in $C^{*}(G)$. If $S$ and $T$ are sets of unitary representations of $G$, then $S$ is weakly contained in $T(S \prec T)$ if

$$
\bigcap_{\sigma \in S} \operatorname{ker} \sigma \supset \bigcap_{\tau \in T} \operatorname{ker} \tau,
$$

and $S$ and $T$ are said to be weakly equivalent $(S \sim T$ if $S \prec T$ and $T \prec S)$. For a closed subgroup $H$ of $G$ and representations $\pi$ of $G$ and $\tau$ of $H$, we denote by $\pi \mid H$ the restriction of $\pi$ to $H$ and by ind ${ }_{H}^{G} \tau$ the representation obtained by inducing $\tau$ up to $G$. We will use throughout that $\pi \prec \operatorname{ind}_{H}^{G}(\pi \mid H)$ if $G$ is amenable [11]. If $H$ is normal then we have the usual action $(x, \sigma) \rightarrow \sigma^{x}$ of $G$ on representations of $H$, and $G(\sigma)$ will signify the $G$-orbit of $\sigma$. Moreover, $1_{G}$ stands for the trivial 1-dimensional representation of $G$ and $\pi \otimes \rho$ for the tensor product of $\pi$ and $\rho$. We will frequently use that forming tensor products preserves weak containment [10]. That is, $\pi_{1} \prec \pi_{2}$ and $\rho_{1} \prec \rho_{2}$ implies

$$
\pi_{1} \otimes \rho_{1} \prec \pi_{2} \otimes \rho_{2} \text {. }
$$

Also if $H$ is a closed subgroup of $G$ and $\sigma$ and $\tau$ are unitary representations of $G$ and $H$, respectively, then

$$
\sigma \otimes \operatorname{ind}_{H}^{G} \tau=\operatorname{ind}_{H}^{G}(\sigma \mid H \otimes \tau)
$$

(see [17, Section 12] and [11,p. 314]). Finally, the dual space $\hat{G}$ of $G$ is the set of all equivalence classes of irreducible unitary representations of $G$, and $\hat{G}$ carries the Jacobson topology [6]. Recall now that two subgroup $A$ and $B$ of $G$ are regularly related in $G$ in the sense of Mackey [17, p. 127] if there exists a sequence $E_{0}, E_{1}$, $E_{2}, \ldots$ of measurable subsets of $G$ each of which is a union of double cosets $A x B$, $x \in G$, such that $E_{0}$ has Haar measure zero and each double coset not in $E_{0}$ is the intersection of the $E_{j}$ containing it. 
LEMMA 1.1. Let $G$ be a second countable locally compact group, $N$ a closed normal subgroup of $G$, and $H$ an amenable closed subgroup of $G$. Suppose that $N \subset G_{F}$ and $H$ and $N$ are regularly related. If $\pi \in G$ satisfies $\pi \sim \operatorname{ind}_{N}^{G}(\pi \mid N)$, then for any $\tau \in \hat{H}, \pi \prec$ ind $_{H}^{G} \tau$ implies $\pi \mid H \succ \tau$.

Proof. Note first that $\tau \prec \operatorname{ind}_{H \cap N}^{H}(\tau \mid H \cap N)$ since $H$ is amenable. Hence

$$
\pi \prec \operatorname{ind}_{H}^{G} \tau \prec \operatorname{ind}_{H}^{G}\left(\operatorname{ind}_{H \cap N}^{H}(\tau \mid H \cap N)\right)=\operatorname{ind}_{H \cap N}^{G}(\tau \mid H \cap N) .
$$

$H$ being second countable there exists $\sigma \in \widehat{H \cap N}$ such that $\tau \mid H \cap N \sim H(\sigma)$. This shows $\pi \prec \operatorname{ind}_{H \cap N}^{G} \sigma$, and therefore $\pi \mid H \cap N \succ \sigma$ by [14, Theorem 2.5]. As $H$ and $N$ are regularly related, [10, Theorem 5.3$]$ yields

$$
\begin{aligned}
\pi \mid H & \sim \operatorname{ind}_{N}^{G}(\pi \mid N) \mid H \succ \operatorname{ind}_{H \cap N}^{H}(\pi \mid H \cap N) \\
& \succ \operatorname{ind}_{H \cap N}^{H} \sigma \sim \operatorname{ind}_{H \cap N}^{H}(\tau \mid H \cap N) \succ \tau .
\end{aligned}
$$

THEOREM 1.2. Let $G$ be a finitely generated nilpotent discrete group. For $\pi \in \hat{G}$ the following conditions are equivalent:

(i) (FP2) holds for $\pi$, i.e. for every subgroup $H$ of $G$ and $\tau \in \hat{H}, \pi \prec \operatorname{ind}_{H}^{G} \tau$ implies $\pi \mid H>\tau$.

(ii) For each cyclic subgroup $H$ of $G$ and $\tau \in \hat{H}, \pi \prec \operatorname{ind}_{H}^{G} \tau$ implies $\pi \mid H \succ \tau$.

(iii) $\pi \sim \operatorname{ind}_{G_{F}}^{G}\left(\pi \mid G_{F}\right)$

Proof. By Lemma 1.1 it suffices to prove (ii) $\Rightarrow$ (iii). Recall first that there exists a character (in the sense of $[5,22]$ ) $\alpha$ on $G$ such that $\pi$ is weakly equivalent to $\pi_{\alpha}$, the cyclic representation associated to $\alpha$ (see [5, Theorem 2.1]). $G$ being finitely generated, there exist normal subgroups $L$ and $N$ of $G$ and a $G$-invariant linear character $\lambda$ of $L$ with the following properties [13, Corollary 1]:

1) $L \subset N$ and $[N: L]<\infty$;

2) $\alpha \mid L=\lambda$ and $\alpha(x)=0$ for all $x \notin N$.

We claim that $L \subset G_{F}$. To this end notice that $G / G_{F}$ is torsion free (compare [13, p. 98, (ii)]). Thus if $L \notin G_{F}$, we can choose an infinite cyclic subgroup $H$ of $L$ such that $H \cap G_{F}=\{e\}$. Now by [12, Proposition 2.3] $\operatorname{ind}_{H}^{G} \chi \sim \hat{G}$ for every character $\chi \in \hat{H}$. By assumption (ii) this implies $\pi \mid H \sim \hat{H}$, contradicting $\pi \mid H \sim$ $\lambda \mid H$. Finally, since $L \subset G_{F}$ and $[N: L]<\infty$, we get $N \subset G_{F}$. Hence we have seen so far that $\alpha$ vanishes outside $G_{F}$. This shows that

$$
\pi_{\alpha}=\operatorname{ind}_{G_{F}}^{G}\left(\pi_{\alpha \mid G_{F}}\right) \text {. }
$$

Moreover, it is well known that $\pi \mid G_{F} \sim \pi_{\alpha \mid G_{F}}$. Therefore we obtain

$$
\pi \sim \pi_{\alpha} \sim \operatorname{ind}_{G_{F}}^{G}\left(\pi \mid G_{F}\right) .
$$

We don't know whether the above theorems remains true for non-finitely generated nilpotent discrete groups $G$. This seems to be a very difficult problem 
even in situations where some informations about characters are available [5]. In fact, we don't even know the answer when $G$ is 2-step nilpotent.

ReMARKs 1.3. a) Let $G$ be a countable amenable discrete group. Then there is a dense subset $D$ in $\hat{G}$ such that every $\pi \in D$ is weakly equivalent to $\operatorname{ind}_{G_{F}}^{G}\left(\pi \mid G_{F}\right)$ and hence has property (FP2). This can be deduced from a result of Thoma $[22$, Satz 4]. To see this let $\alpha$ be a character on $G$ vanishing off $G_{F}$. Then $\pi_{\alpha} \sim \operatorname{ind}_{G_{F}}^{G}\left(\pi_{\alpha} \mid G_{F}\right)$, and $\pi_{\alpha}$ is a factor representation. Now, since $C^{*}(G)$ is separable, kernels of factor representations of $C^{*}(G)$ are primitive ideals $[6,(3.9 .1)$ and (5.7.6)]. Thus $\pi_{\alpha} \sim \rho_{\alpha}$ for some $\rho_{\alpha} \in \hat{G}$.

b) Suppose that $G$ is a finitely generated torsion free nilpotent discrete group, and let $\pi \in \hat{G}$. If $\pi \mid Z(G)$ is faithful, then $\pi$ satisfies (FP2). Indeed, there is a character $\alpha$ on $G$ with $\pi \sim \pi_{\alpha}$, and it suffices to notice that $\alpha$ vanishes off $Z(G)$. As $G$ is torsion free, $G_{F}=Z(G)$, and since $\alpha \mid Z(G)$ is faithful, $\alpha \mid Z_{2}(G) \backslash Z_{1}(G)=0$ (see [5,13]). These two facts imply $\alpha \mid G \backslash Z(G)=0[13$, Lemma 1].

c) As an example, consider the group $G$ of all integer upper triangular $d \times d$ matrices. Using b) it is easily seen that (FP2) holds for $\pi \in \hat{G}$ if and only if $\pi \mid Z(G)$ is faithful.

\section{Connected Nilpotent Groups.}

Let $G$ be a connected nilpotent group and $\pi \in \hat{G}$. In general, $\pi$ need not share property (FP1), yet it does provided that it is square integrable modulo its kernel [2]. The purpose of this section is to give, under the same assumption on $\pi$, a criterion for $\pi$ to satisfy (FP2).

Nielsen's papers [19] and [20] are concerned with the extent to which (FPC2) fails to hold for a connected and simply connected nilpotent Lie group. The following Theorem 2.1 is due to him and will substantially be used in the sequel. As Nielsen does not give a proof but only points out that his methods can be used to show it [19, p. 309], we include a fairly short proof for the readers convenience.

THEOREM 2.1 [19]. If $G$ is a simply connected nilpotent Lie group, then for every non-normal 1-dimensional subgroup $H$ of $G$ and $\alpha \in \widehat{H}$,

$$
\operatorname{ind}_{H}^{G} \alpha \succ 1_{G} \text {. }
$$

Proof. We first assume $H \subset Z_{2}(G)$ and $\operatorname{dim} Z(G)=1 . N=H Z(G)$ is a closed $[2$, Lemma 1.1] abelian normal subgroup of $G$. Now,

$$
\operatorname{ind}_{H}^{N} \alpha \sim\{\alpha\} \times \widehat{Z(G)},
$$

and it suffices to show that $1_{N}$ is weakly contained in the $G$-orbit of $\operatorname{ind}_{H}^{N} \alpha$, since then

$$
\operatorname{ind}_{H}^{G} \alpha \succ \operatorname{ind}_{N}^{G} 1_{N} \succ 1_{G}
$$


Thus we have to find $\gamma_{n} \in \widehat{Z(G)}$ and $x_{n} \in G, n \in \mathrm{N}$, such that $\left(\alpha \times \gamma_{n}\right)^{x_{n}} \rightarrow 1_{N}$ in $\widehat{N}$. Now, for $x \in G, y \in H, z \in Z(G)$, and $\gamma \in \widehat{Z(G)}$,

$$
(\alpha \times \gamma)^{x}(y z)=\alpha(y) \gamma\left(\left[y^{-1}, x^{-1}\right]\right) \gamma(z) .
$$

$y \rightarrow \gamma\left(\left[y^{-1}, x^{-1}\right]\right)$ defines a character $\gamma_{x}$ of $H$, and in fact $\Phi: x \rightarrow \gamma_{x}$ is a continuous homomorphism of $G$ into $\widehat{H}=\mathrm{R}$. If $\gamma \neq 1_{Z(G)}$, then $\gamma_{x} \neq 1_{H}$ for some $x \in G$ because otherwise the connected set $[H, G]$ would be contained in the discrete kernel of $\gamma$, and hence $H \subset Z(G)$. It follows that $\Phi(G)$, being a connected subgroup of $\widehat{H}$, coincides with $\widehat{H}$. Therefore, if we choose any sequence $\gamma_{n} \in \widehat{Z(G)}$ with $\gamma_{n} \rightarrow 1_{Z(G)}$ and $\gamma_{n} \neq 1_{Z(G)}$, then for every $n$ there exists $x_{n} \in G$ such that $\left(\gamma_{n}\right)_{x_{n}}=\bar{\alpha}$. Hence

$$
\left(\alpha \times \gamma_{n}\right)^{x_{n}}=1_{H} \times \gamma_{n} \rightarrow 1_{N} .
$$

Next we drop the hypothesis $\operatorname{dim} Z(G)=1$ and argue by induction on $d=\operatorname{dim} G$. If $d=3$, the smallest possible dimension for a non-abelian $G$, then $\operatorname{dim} Z(G)=1$. Suppose $d \geqq 4$ and $\operatorname{dim} Z(G) \geqq 2$, the case $\operatorname{dim} Z(G)=1$ being dealt with above. Then, for some connected subgroup $V \neq 0$ of $Z(G), H$ is non-central, and hence non-normal, modulo $V$. Let $q$ : $G \rightarrow \dot{G}=G / V$ and $\dot{H}=H V / V$. By induction hypothesis, $\operatorname{ind}_{\mathscr{H}}^{\dot{G}} \dot{\alpha} \succ 1_{\dot{G}}$ for each $\dot{\alpha} \in \hat{H}$, and hence

$$
1_{G} \prec\left(\operatorname{ind}_{H}^{\dot{G}} \dot{\alpha}\right) \circ q=\operatorname{ind}_{H V}^{G}(\dot{\alpha} \circ q) \prec \operatorname{ind}_{H}^{G}(\dot{\alpha} \circ q \mid H) \text {. }
$$

As $\hat{H} \circ(q \mid H)=\widehat{H}$ this finishes the proof of the theorem under the assumption $H \subset Z_{2}(G)$.

Finally, for the general case, let $k$ be minimal with $H \subset Z_{k+1}(G)$, and assume $k \geqq 2$. Let $q: G \rightarrow \dot{G}=G / Z_{k-1}(G)$ and $\dot{H}=q(H)$. Then $\dot{H} \subset Z_{2}(\dot{G})$, and $\dot{H}$ is non-central in $\dot{G}$ since $H \notin Z_{k}(G)$. By what we have shown so far, ind $\dot{G} \dot{\alpha} \succ 1_{G}$ for every $\alpha \in \hat{H}$, and this implies $1_{G} \prec$ ind $_{H}^{G} \alpha$ for all $\alpha \in \widehat{H}$ by the same argument as above.

We will need in the proofs of Lemma 2.2 and Theorem 2.5 that if $G$ is a connected nilpotent group and $K$ a compact normal subgroup of $G$, then $K$ is contained in the center of $G$. This is certainly known to experts and can be seen by fairly standard arguments. We nevertheless include these for the readers convenience. Let $K_{j}=K \cap Z_{j}(G), j=1,2, \ldots$ It suffices to show that $K_{j} \subset Z(G)$ implies $K_{j+1} \subset Z(G)$. For that, take any $a \in K_{j+1}$ and denote by $L$ the closed subgroup of $G$ generated by $K_{j}$ and $a$. Then $L$ is abelian as $K_{j} \subset Z(G)$, and $L$ is normal in $G$ since $L / K_{j}$ is contained in the center of $G / K_{j}$. Now, since $\hat{L}$ is discrete and $G$ is connected, for every $\lambda \in \hat{L}$ the $G$-orbit $G(\lambda)$ consists only of $\lambda$. Thus each character of $L$ is $G$-invariant, and this proves $L \subset Z(G)$.

LEMMA 2.2. The center of a connected nilpotent group is connected. 
ProOF. We first consider a connected nilpotent Lie group $G$. Let $\tilde{G}$ be a simply connected covering group of $G$ and $p: \tilde{G} \rightarrow G$ a covering homomorphism. Then $Z(\tilde{G})=p^{-1}(Z(G))$, and $Z(\tilde{G})$ is a . Thus $Z(G)$ is connected.

Now let $G$ be a connected nilpotent group. $G$ is a projective limit of Lie groups $G_{\imath}=G / K_{\imath}, \imath \in I$. Fix a compact normal subgroup $K$ of $G$ such that $G / K$ is Lie. By what we noticed above, $K$ is contained in the center of $G$. We are going to show that for any such $K, Z(G) / K$ is connected. From this it follows readily that $Z(G)$ is connected. Of course, we can assume $K_{\iota} \subset K$ for all $\iota \in I$. Let $Z=Z(G)$ and

$$
Z_{\imath}=\left\{x \in G: x K_{l} \in Z\left(G / K_{\imath}\right)\right\} \text {. }
$$

Then $Z_{l} / K$ is connected by the first paragraph of the proof. For any locally compact group $F$ denote by $\mathscr{S}(F)$ the set of all closed subgroups of $F$. Recall Fell's topology [9] which makes $\mathscr{S}(F)$ a compact Hausdorff space. A subbasis is given by the sets

$$
U(C, V)=\{A \in \mathscr{S}(F) ; A \cap C=/ \quad Q\}, A \cap V \mid
$$

where $C$ is compact and $V$ is open in $F$. We can assume that $Z_{t} \rightarrow Z_{0}$ in $\mathscr{S}(G)$. It is easily verified that $Z_{0}=Z$. Since $K$ is compact, we conclude that $Z_{t} / K \rightarrow Z / K$ in $\mathscr{S}(G / K)$. Now, all these groups are contained in $Z(G / K)$, and $Z(G / K)$ is a connected Lie group, hence of the form $V \times C$ where $V$ is a vector group and $C$ a compact connected Lie group. Since $Z_{l} / K$ is connected, $Z_{t} / K=V_{l} \times \mathrm{C}_{l}$, where $C_{l}$ is a closed connected subgroup of $C$ and $V_{l}$ a vector subgroup of $Z(G / K)$. But notice that $V_{1}$ need not be contained in $V$. Next, we can moreover assume that $C_{t} \rightarrow C_{0}$ in $\mathscr{S}(C)$. Notice that necessarily $C_{0} \subset C_{t_{0}}$ for every $\imath_{0} \in I$. Indeed, if $K_{t_{2}} \subset K_{t_{1}}$, then $Z_{l_{2}} \subseteq Z_{l_{1}}$ and hence $C_{l_{2}} \subset C_{l_{1}}$, and $x \in C_{0}$ if and only if there exists a net $x_{l}, l \in I$, such that $x_{l} \rightarrow x$ and $x_{t} \in C_{l}$. It follows that $C_{l} / C_{0} \rightarrow\left\{C_{0}\right\}$ in $\mathscr{S}\left(C / C_{0}\right)$. Now the Lie group $C / C_{0}$ does not contain arbitrarily small subgroups. Therefore we can assume $C_{\imath}=C_{0}$ for all $\imath \in I$. Moreover, $C_{0}$ is the maximal compact subgroup of $\dot{Z}=Z / K$. It remains to show that $\dot{Z} / C_{0}$ is connected.

Let $p$ denote the projection $Z(G / K)=V \times C \rightarrow V$. Then $p$ maps $V_{l}, l \in I$, and $\dot{Z} / C_{0}$ homeomorphically onto $p\left(V_{l}\right)$ and $p\left(\dot{Z} / C_{0}\right)$, respectively. Since $C$ is compact, $p\left(V_{l}\right) \rightarrow p\left(\dot{Z} / C_{0}\right)$ in $\mathscr{S}(V)$. Finally, $V$ and $p\left(V_{l}\right), \imath \in I$, are s, and the limit of a convergent net of $\mathrm{s}$ must be a . In particular, $p\left(\dot{Z} / C_{0}\right)$ is connected.

Let $\pi$ be a representation of the locally compact group $G$ in the Hilbert space $\mathscr{H}_{n}$, and denote by $I_{\pi}$ the identity operator on $\mathscr{H}_{\pi}$. The kernel $K$ and the projective kernel $P$ of $\pi$ are defined as

$$
K=\left\{x \in G ; \pi(x)=I_{\pi}\right\} \text { and } P=\left\{x \in G ; \pi(x)=\lambda(x) I_{\pi} \text { for some } \lambda(x) \in \mathrm{T}\right\},
$$

respectively. Suppose now that $\pi$ is irreducible. Then $P / K$ coincides with the center of $G / K$. In particular, $\lambda$ is a $G$-invariant character on $P$. 
COROLlary 2.3. Let $G$ be a connected nilpotent group and $\pi$ an irreducible representation of $G$. Then the projective kernel $P$ of $\pi$ is connected.

Proof. Let $K_{0}$ be the connected component of the kernel $K$ of $\pi$. By passing over to $G / K_{0}$, we can assume that $K$ is totally disconnected. We claim that $P=Z(G)$. For $x \in P$ and $y \in G$,

$$
\pi([x, y])=\pi(x) \pi(y) \pi(x)^{-1} \pi(y)^{-1}=I_{\pi},
$$

so that $[x, G]=\{[x, y] ; y \in G\} \subset K$. Since $[x, G]$ is connected, we obtain $[x, G]=\{e\}$. This shows $P=Z(G)$ which is connected by Lemma 2.2.

Retain the notations used above, and let $\pi$ be irreducible. Then $\pi$ is called square integrable modulo its kernel if every coordinate function

$$
x \rightarrow\langle\pi(x) \xi, \eta\rangle, \xi, \eta \in \mathscr{H}_{\pi},
$$

(being constant on cosets of $K$ ) is square integrable on $G / K$.

Let us briefly review Kirillov's theory. Suppose that $G$ is a connected and simply connected nilpotent Lie group with Lie algebra g. Denote by $\mathrm{Ad}^{*}$ the coadjoint representation of $G$ on $\mathfrak{g}^{*}$, the dual vector space of $\mathfrak{g}$. Kirillov [16] established a mapping $f \rightarrow \pi_{f}$ from $\mathrm{g}^{*}$ onto $\hat{G}$ such that for $f, g \in \mathrm{g}^{*}, \pi_{f}=\pi_{g}$ if and only if $g \in \operatorname{Ad}^{*}(G) f$. The Kirillov correspondence $\mathrm{g}^{*} / \mathrm{Ad}^{*}(G) \rightarrow \hat{G}$ is a homeomorphism provided that the orbit space $\mathrm{g}^{*} / \operatorname{Ad}^{*}(G)$ is endowed with the quotient topology [4]. The coadjpint orbits are closed in $\mathrm{g}^{*}$, and $\pi_{f} \in \hat{G}$ is square integrable modulo its kernel if and only if $\operatorname{Ad}^{*}(G) f$ is a linear variety [3, Theorem 1.1].

It is worth calling attention to the following facts which, for instance, can be found in [7, p. 284]. These facts reduce weak containment questions to orbit geometry and certainly make our arguments in Sections 3 and 4 more transparent.

Let $\mathfrak{h}$ be a subalgebra $\mathfrak{g}$ and $H=\exp \mathfrak{h}$, and let $p: \mathfrak{g}^{*} \rightarrow \mathfrak{h}^{*}$ be the natural map. Let $\pi \in \hat{G}$ and $\tau \in \hat{H}$ with coadjoint orbits $O_{\pi}$ and $O_{\tau}$ in $\mathrm{g}^{*}$ and $\mathfrak{h}^{*}$, respectively. Then

(i) $\pi \mid H \succ \tau$ if and only $O_{\tau} \subset \overline{p\left(O_{\pi}\right)}$;

(ii) $\pi \prec \operatorname{ind}_{H}^{G} \tau$ if and only if $O_{\pi} \subset \overline{\operatorname{Ad}^{*}(G)\left(p^{-1}\left(O_{\tau}\right)\right)}$.

LEMMA 2.4. Let $G$ be a connected nilpotent group and $\pi \in \hat{G}$, and suppose that $\pi$ is square integrable modulo its kernel. Then

$$
\pi \sim \operatorname{ind}_{P}^{G}(\pi \mid P) \text {. }
$$

Proof. Let $q: G \rightarrow \dot{G}=G / K$, and let $\pi \in \hat{G}$ be defined by $\pi \circ q=\pi$. $\pi$ is square integrable, hence an open point in $\hat{G}$ by [1, Theorem 3]. Suppose that we have shown $\pi \sim \operatorname{ind}_{Z(\dot{G})}^{\dot{G}}(\pi \mid Z(\dot{G}))$. Then, since $P=q^{-1}(Z(\dot{G}))$,

$$
\left.\pi \sim \operatorname{ind}_{Z(\dot{G})}^{G}(\pi \mid Z(\dot{G})) \circ q=\operatorname{ind}_{P}^{G}(\pi \mid Z(\dot{G})) \circ q\right)=\operatorname{ind}_{P}^{G}(\pi \mid P) .
$$


Thus we can assume that $\pi$ is faithful. It follows that $G$ is a Lie group. In fact, $G$ is a projective limit of Lie groups $G / K_{\imath}, \imath \in I$, and

$$
\hat{G}=\cup_{i \in I} \widehat{G / K},[18 \text {, Proposition 2.2]. }
$$

Let $p: \tilde{G} \rightarrow G$ be a simply covering of $G$. Then $Z(\tilde{G})=p^{-1}(Z(G))$, and $\pi \circ p \in \hat{\widetilde{G}}$ is an open point in

$$
R=\{\rho \in \hat{\widetilde{G}} ; \rho|Z(\tilde{G}) \sim \pi \circ p| Z(\tilde{G})\} .
$$

From this we have to conclude $\pi \circ \operatorname{ind}_{Z(\tilde{G})}^{\tilde{G}}(\pi \circ p \mid Z(\tilde{G}))$. Therefore, in terms of Kirillov's theory, we are reduced to the following observation. If $G=\exp g$ is simply connected nilpotent, and $z$ the center of $g$ and $f \in \mathfrak{g}^{*}$ is such that $\operatorname{Ad}^{*}(G) f$ is open in $f+z^{\perp}$, then $\operatorname{Ad}^{*}(G) f=f+z^{\perp}$. But this is clear since $\operatorname{Ad}^{*}(G) f$ is also closed.

The statement of Lemma 2.4 is equivalent to saying that $\operatorname{ind}_{P}^{G}(\pi \mid P)$ is a multiple of $\pi$. This is due to the fact that connected nilpotent groups are CCR, that is, points in $\hat{G}$ are closed. Indeed, if $\pi \in \hat{G}$, then $\pi \in \hat{H}$ for some Lie quotient $H=G / K$ (compare the proof of Lemma 2.4), and $H$ is CCR since its simply connected covering group is.

Continue to consider a connected nilpotent group $G$, and denote by $G^{c}$ the set of all compact elements in $G$. That is, $x \in G^{c}$ if and only if the closed subgroup of $G$ generated by $x$ is compact. We claim that $G^{c}$ is a compact and connected normal subgroup of $G$. Once this is shown, $G^{c}$ is the unique maximal compact subgroup of $G$. In particular, by the remark preceding Lemma $2.2, G^{c}$ is contained in the center of $G$. The above claim being true for connected abelian groups, we now proceed to verify it by induction on the length of nilpotency of $G$. Thus assume that $(G / Z(G))^{c}$ is a compact and connected (normal) subgroup of $G / Z(G)$, and let

$$
H=\left\{x \in G ; x Z(G) \in(G / Z(G))^{c}\right\}
$$

Then $H^{c}=G^{c}$, and $H$ is connected since $H / Z(G)$ and $Z(G)$ are connected (Lemma 2.2). By the Freudenthal-Weil theorem [6,(16.4.6)], $H=V \times K$ where $K$ is compact connected and $V$ is a . Obviously $K=H^{c}=G^{c}$, and this gives the desired result.

Recall next that, for any locally compact group $G, G_{F}$ denotes the normal subgroup of $G$ consisting of all elements in $G$ with relatively compact conjugacy classes. Tits [23] has shown that $G_{F}$ is closed provided that $G$ is connected. Suppose again that $G$ is connected and nilpotent. For such $G, G_{F}$ is easy to describe. In fact, denoting by $C$ the maximal compact subgroup of $G$, we have $(G / C)_{F}=G_{F} / C$, and $G / C$ is simply connected nilpotent. Hence $(G / C)_{F}$ coincides 
with the center of $G / C$. Thus

$$
G_{F}=q^{-1}(Z(G / C)),
$$

where $q: G \rightarrow G / C$ denotes the quotient homomorphism.

THEOREM 2.5. Let $G$ be a connected nilpotent group and $\pi \in \hat{G}$, and suppose that $\pi$ is square integrable modulo its kernel. Then the following conditions are equivalent:

(i) $\pi$ satisfies (FP2).

(ii) $\pi$ satisfies (FPC2).

(iii) $\pi \sim \operatorname{ind}_{G_{F}}^{G}\left(\pi \mid G_{F}\right)$.

ProOF. The implication (iii) $\Rightarrow$ (i) is an immediate consequence of Lemma 1.1 as soon as we know that for any closed subgroup $H$ of $G, H$ and $G_{F}$ are regularly related. Since $H x G_{F}=H G_{F} x, x \in G$, it suffices to observe that $H G_{F}$ is closed in $G$. To verify this, let $C$ be as above. Then

$$
H G_{F} / C=H C / C \cdot G_{F} / C=H C / C \cdot Z(G / C),
$$

which is closed in $G / C$ by [2, Lemma 1.1]. Hence $H G_{F}$ is closed in $G$.

To prove (ii) $\Rightarrow$ (iii), we consider the projective kernel $P$ of $\pi$. By Lemma 2.4,

$$
\pi \sim \operatorname{ind}_{P}^{G}(\pi \mid P) .
$$

Suppose that we already know $P \subset G_{F}$. Then

$$
\pi \mid G_{F} \prec \operatorname{ind}_{P}^{G_{F}}(\pi \mid P),
$$

since $G_{F}$, being a closed subgroup of a nilpotent group, is amenable. Hence, by induction in stages and since inducing preserves weak containment,

$$
\operatorname{ind}_{G_{F}}^{G}\left(\pi \mid G_{F}\right) \prec \operatorname{ind}_{G_{F}}^{G}\left(\operatorname{ind}_{G}^{G_{F}}(\pi \mid P)\right)=\operatorname{ind}_{P}^{G}(\pi \mid P) \sim \pi .
$$

On the other hand $\pi \prec \operatorname{ind}_{G_{F}}^{G}\left(\pi \mid G_{F}\right)$ again by amenability of $G$. Thus it remains to show $P \subset G_{F}$.

Clearly, the maximal compact subgroup $C$ of $G$ is contained in $P$ as $C$ is central in $G$. We are going to show that $P / C \subset Z(G / C)$. Otherwise, since $P / C$ is connected (Corollary 2.3 ) and $G / C$ is simply connected, there exists a 1-dimensional connected subgroup $H$ of $P / C$ which is non-central in $G / C$, and hence non-normal. By Theorem 2.1,

$$
1_{G / C} \prec \operatorname{ind}_{H}^{G / C} \alpha
$$

for every $\alpha \in \hat{H}$. Therefore, with $q: G \rightarrow G / C$,

$$
1_{G} \prec\left(\operatorname{ind}_{H}^{G / C} \alpha\right) \circ q=\operatorname{ind}_{q^{-1}(H)}^{G}(\alpha \circ q) .
$$


Now $q^{-1}(H) \subset P$ and $\pi \mid P \sim \lambda$ for some $G$-invariant character $\lambda$ of $P$. Thus

$$
\begin{aligned}
\pi \prec \pi \otimes \operatorname{ind}_{q^{-1}(H)}^{G}(\alpha \circ q) & =\operatorname{ind}_{q^{-1}(H)}^{G}\left(\pi \mid q^{-1}(H) \otimes \alpha \circ q\right) \\
& \sim \operatorname{ind}_{q^{-1}(H)}^{G}\left(\lambda \mid q^{-1}(H) \otimes \alpha \circ q\right) .
\end{aligned}
$$

Since (FPC2) holds for $\pi$ and $q^{-1}(H)$ is connected as $H$ and $C$ are, we obtain

$$
\pi\left|q^{-1}(H) \succ \lambda\right| q^{-1}(H) \otimes \alpha \circ q
$$

for all $\alpha \in \hat{H}$, a contradiction.

COROLlaRY 2.6. Let $G$ be a simply connected nilpotent Lie group and $Z$ its center. Let $\pi \in \hat{G}$, and suppose that the Kirillov orbit corresponding to $\pi$ is a linear variety. Then the following are equivalent:

(i) $\pi$ has the Frobenius property (FP2).

(ii) For each 1-dimensional connected subgroup $H$ of $G$ and $\tau \in \hat{H}, \pi \prec \operatorname{ind}_{H}^{G} \tau$ implies $\pi \mid H \succ \tau$.

(iii) $\pi \sim \operatorname{ind}_{Z}^{G}(\pi \mid Z)$.

ProOF. (ii) $\Rightarrow$ (iii) is obvious from the proof of Theorem 2.5 since $C$ is trivial.

It is worth pointing out that in the Lie algebra context (iii) of Corollary 2.6 reads as $\operatorname{Ad}^{*}(G) f=f+z^{\perp}$, where $\pi=\pi_{f}, f \in \mathfrak{g}^{*}$. Recall also that all the coadjoint orbits in $\mathrm{g}^{*}$ are linear varieties if $G$ is 2 -step nilpotent. However, the implication (i) $\Rightarrow$ (iii) doesn't remain true in general once we drop the assumption on $\pi$. In fact, in Section 3 we will exhibit a series of simply connected nilpotent Lie groups each of which possesses sufficiently many irreducible representations to carry Plancherel measure which share property (FP2) and nevertheless violate (iii). These representations will even be induced from characters of abelian normal subgroups. We conclude this section with the following lemma which in special situations considerably simplifies verifying (FP2) or (FPC2) for a given representation.

LEMMA 2.7. Let $G$ be a simply connected nilpotent Lie group and $H$ a closed subgroup of $G$. Let $\pi \in \hat{G}$ such that $\tau \sim \operatorname{ind}_{N}^{G} \lambda$, where $N$ is a connected normal subgroup of $G$ and $\lambda \in \hat{H}$. Suppose that for every $\sigma \in \widehat{H \cap N}, \pi \prec \operatorname{ind}_{H \cap N}^{G} \sigma$ implies $\pi \mid H \cap N \succ \sigma$. Then, for each $\tau \in \hat{H}, \pi \prec \operatorname{ind}_{H}^{G} \tau$ implies $\pi \mid H \succ \tau$.

Proof. As $H$ is second countable, $\tau \mid H \cap N \sim H(\sigma)$ for some $\sigma \in \widehat{H \cap N}$, and hence

$$
\pi \prec \operatorname{ind}_{H}^{G} \tau \prec \operatorname{ind}_{H \cap N}^{G}(\tau \mid H \cap N) \sim \operatorname{ind}_{H \cap N}^{G} \sigma .
$$

Therefore, $\pi \mid H \cap N>\sigma$, and this shows

$$
\pi|H \cap N>H(\sigma) \sim \tau| H \cap N .
$$


On the other hand, $\pi|H \cap N \sim G(\lambda)| H \cap N$ and $H$ and $N$ are regularly related in $G$ (compare the proof of Theorem 2.5). Thus [9, Theorem 5.3] yields

$$
\begin{gathered}
\pi \mid H \sim\left\{\operatorname{ind}_{H \cap N}^{H}\left(\lambda^{x} \mid H \cap N\right) ; x \in G\right\} \sim \operatorname{ind}_{H \cap N}^{H}(\pi \mid H \cap N) \\
>\operatorname{ind}_{H \cap N}^{H}(\tau \mid H \cap N) \succ \tau .
\end{gathered}
$$

\section{Nilpotent Groups of the Form $R \bowtie R^{m}$.}

Among all simply connected nilpotent Lie groups those which are in addition semi-direct products of $R$ with $R^{m}$ form a fairly accessible subclass. In [2] the Frobenius properties (FP1) and (FPC1) have been studied for such $G$, and our concern here is to investigate (FP2) and (FPC2).

For each $d \in \mathrm{N}, d \geqq 2$, let $\mathrm{g}_{d}$ denote the $(d+1)$-dimensional Lie algebra with generators $X, X_{d}, \ldots, X_{1}$ and non-trivial Lie products $\left[X, X_{j}\right]=X_{j-1}$, $2 \leqq j \leqq d$. These algebras are called threadlike. $g_{d}$ is $d$-step nilpotent and a semidirect product of $\mathrm{R} X$ with the abelian ideal $\sum_{j=1}^{d} \mathrm{R} X_{j}$. In what follows we will always identify $\sum_{j=1}^{d} R X_{j}$ with $\exp \left(\sum_{j=1}^{d} R X_{j}\right)$ and with $\mathrm{R}^{d} . G_{d}=\exp g_{d}$ is a semi-direct product of $R$ with $R^{d}$. Conversely, every nilpotent Lie algebra $\mathfrak{g}$ containing an abelian ideal of codimension one can be built up from these $\boldsymbol{g}_{d}$. Studying topological Frobenius properties for $G=\exp g$ can be reduced to the case of a direct product of some $G_{d}$ with a vector group.

Lemma 3.1. Let $G=G_{d} \times V$, where $V$ is a vector group and $G_{d}$ is as above. Suppose that $H$ is a closed subgroup of $\mathrm{R}^{d} \times V$ such that $H_{0}$, the connected component of $H$, is contained in $\mathrm{R} X_{1} \times V$, the center of $G$. Then for $\pi \in \hat{G} \backslash G / R X_{1}$,

$$
\pi \mid H>\{\gamma \in \hat{H} ; \gamma|H \cap Z(G) \sim \pi| H \cap Z(G)\} .
$$

Proof. For simplicity we write the abelian normal subgroup $N=R^{d} \times V$ additively. Since $H_{0} \subset Z(G)=\mathrm{R} X_{1}+V$, there exists a lattice

$$
\Gamma=Z Y_{2}+\ldots+Z Y_{d}
$$

in $\mathrm{R}^{d-1}$ such that $H \subset \Gamma+Z(G)$. Let $F=\Gamma+Z(G), \pi \mid Z(G) \sim \lambda \in \widehat{Z(G)}$ and

$$
Y_{k}=\sum_{j=2}^{d} a_{k j} X_{j}, a_{k j} \in \mathrm{R}, 2 \leqq k, j \leqq d .
$$

Once we have shown $\pi \mid F \sim \hat{\Gamma} \times\{\lambda\}$, it follows that

$$
\pi \mid \hat{H} \sim\{\beta \in \hat{H} ; \beta|H \cap Z(G) \sim \lambda| H \cap Z(G)\} .
$$

Indeed, for fixed $\gamma_{0} \in \hat{\Gamma}, \hat{\Gamma} \otimes\{\lambda\}=(\gamma \times \lambda) \cdot \widehat{F / Z(G)}$, and hence

$$
\left(\hat{\Gamma} \times\{\lambda\}\left|H=\left(\gamma_{0} \times \lambda\right)\right| H \cdot \widehat{F / Z(G)} \mid H,\right.
$$


and $\widehat{F / Z(G)} \mid H$ is a subgroup of $(H / H \cap Z(G))^{\wedge}$ that separates the points and therefore is dense in $(H / H \cap Z(G))^{\wedge}$.

Let $f, f_{d}, \ldots, f_{1}$ denote the basis of $\mathrm{g}_{d}^{*}$ dual to $X, X_{d}, \ldots, X_{1}$, and let $g=c f+$ $\sum_{j=1}^{d} c_{j} f_{j}+h, h \in V^{*}$, with $\pi_{g}=\pi$. Notice that $\lambda\left(s X_{1}\right)=\exp \left(2 \pi i c_{1} s\right)$ for $s \in \mathrm{R}$ and that $c_{1} \neq 0$ as $\pi \notin \widehat{G / R X_{1}}$. The elements $Y \in F$ are of the form

$$
Y=s X_{1}+\sum_{k=2}^{d} n_{k} Y_{k}+v, v \in V, s \in \mathrm{R}, n_{k} \in \mathrm{Z}, 2 \leqq k \leqq d .
$$

We obtain for $t \in \mathbf{R}$

$$
\begin{gathered}
\operatorname{Ad}(\exp t X) g Y)=c_{1} f_{1}(Y)=\sum_{m=2}^{d} f_{m}(Y)\left(\sum_{j=0}^{m-1}(-1)^{j} c_{m-j} \frac{t^{j}}{j !}\right)+h(v) \\
=c_{1} s+\sum_{m=2}^{d}\left(\sum_{j=0}^{m-1}(-1)^{j} c_{m-j} \frac{t^{j}}{j !}\right)\left(\sum_{k=2}^{d} n_{k} a_{k m}\right)+h(v) \\
=c_{1} s+\sum_{k=2}^{d} n_{k} p_{k}(t)+h(v)
\end{gathered}
$$

where the polynomials $p_{k}, 2 \leqq k \leqq d$ are defined by

$$
p_{k}(t)=\sum_{m=2}^{d} a_{k m}\left(\sum_{j=0}^{m-1}(-1)^{j} c_{m-j} \frac{t^{j}}{j !}\right)
$$

Since the polynomials $q_{m}(t)=\sum_{j=0}^{m-1}(-1)^{j} c_{m-j} \frac{t^{j}}{j !}, 2 \leqq m \leqq d$, are of strictly increasing degrees and the matrix $\left(a_{k m}\right) \in M(d-1, \mathrm{R})$ is non-singular, the nonconstant polynomials $p_{2}(t), \ldots, p_{d}(t)$ are linearly independent. By a uniform distribution theorem of Weyl [24, Satz 8] the set

$$
\left\{\left(p_{2}(t), \ldots, p_{d}(t)\right) ; t \in \mathrm{R}\right\}
$$

is dense in $[0,1]^{d-1}$ modulo $Z^{d-1}$. It follows that $\pi \mid F \sim \hat{\Gamma} \times\{\lambda\}$.

LEMMA 3.2. Let $G$ and $\pi$ be as in Lemma 3.1. Suppose that $H$ is a closed subgroup of $\mathrm{R}^{d}+V$ such that $H_{0}$ is not contained in $\mathrm{R} X_{1}+V$. Then, for any $\tau \in \widehat{H}$, $\pi \prec \operatorname{ind}_{H}^{G} \tau$ implies $\pi \mid H \succ \tau$.

PROOF. In what follows we use the abbreviation $e(x)=\exp (2 \pi i x)$ for $x \in \mathrm{R}$. Since $H_{0} \notin \mathrm{R} X_{1}+V$ we can choose $A=\sum_{k=1}^{d} a_{k} X_{k}+v \in \mathrm{R}^{d}+V, v \in V$, with $\mathrm{R} A \subset H$ and $a_{k} \neq 0$ for some $k \geqq 2$. Let $m$ denote the maximal such $k$. Next choose $f \in \mathfrak{g}^{*}$ and $h \in \mathfrak{h}^{*}$ such that $\pi_{f}=\pi$ and $\tau(Y)=e(h(Y))$ for all $Y \in H$. As $\pi \prec \operatorname{ind}_{H}^{G} \tau$, there exist $f_{n} \in \mathfrak{g}^{*}$ and $x_{n} \in G, n \in \mathrm{N}$, such that

$$
f_{n} \mid \mathfrak{h} \in O_{\tau}=\{h\} \text { and } \operatorname{Ad}^{*}\left(x_{n}\right) f_{n} \rightarrow f \text {. }
$$


Now, $G$ being a semi-direct product of $\exp \mathrm{R} X$ with a vector group $N$, we can write

$$
\operatorname{Ad}^{*}\left(x_{n}\right) f_{n}=\operatorname{Ad}^{*}\left(\exp t_{n} X\right)\left(\operatorname{Ad}^{*}\left(y_{n}\right) f_{n}\right)
$$

for certain $t_{n} \in \mathrm{R}$ and $y_{n} \in N$. Since $N$ is abelian, we obtain with $g_{n}=\operatorname{Ad}^{*}\left(y_{n}\right) f_{n}$ :

1) $\operatorname{Ad}^{*}\left(\exp t_{n} X\right) g_{n} \rightarrow f$ in $\mathfrak{g}^{*}$.

2) $e\left(g_{n}(Y)\right)=\tau(Y)$ for all $Y \in H$.

Notice that $g_{n}(A)=h(A)$. In fact, $g_{n}(0)=h(0), e\left(g_{n}(s A)\right)=e(h(s A))$ for all $s \in \mathrm{R}$, and $s \rightarrow g_{n}(s A)-h(s A)$ is continuous. As before denote by $f_{1}, \ldots, f_{d}$ the basis of $\left(\mathrm{R}^{d}\right)^{*}$ dual to $X_{1}, \ldots, X_{d}$. Let

$$
f \mid \mathbf{R}^{d}+V=\sum_{k=1}^{d} r_{k} f_{k}+l \text { and } g_{n} \mid \mathbf{R}^{d}+V=\sum_{k=1}^{d} s_{n, k} f_{k}+l_{n},
$$

$l, l_{n} \in V^{*}$. (1) now implies equations

$$
s_{n, k}=r_{k}+\varepsilon_{n, k}+\sum_{j=1}^{k-1} \frac{(-1)^{j-1}}{j !} s_{n, k-j} t_{n}^{j},
$$

where for each $1 \leqq k \leqq d, \varepsilon_{n, k} \rightarrow 0$ as $n \rightarrow \infty$. Using (3) it is easily verified by induction on $k$ that

$$
s_{n, k}=\frac{1}{(k-1) !} s_{n, 1} t_{n}^{k-1}+\sum_{j=0}^{k-2} \frac{1}{j !}\left(r_{k-j}+\varepsilon_{n, k-j}\right) t_{n}^{j} .
$$

From (4) and $h(A)=g_{n}(A)$ we obtain

$$
\begin{aligned}
& h(A)=\sum_{k=1}^{d} s_{n, k} f_{k}(A)+l_{n}(v)=\sum_{k=1}^{m} a_{k} s_{n, k}+l_{n}(v)= \\
& \frac{a_{m}}{(m-1) !} s_{n, 1} t_{n}^{m-1}+s_{n, 1} \sum_{k=1}^{m-1} \frac{a_{k}}{(k-1) !} t_{n}^{k-1}+ \\
& \sum_{k=1}^{m} a_{k}\left(\sum_{j=0}^{k-2} \frac{1}{j !}\left(r_{k-j}+\varepsilon_{n, k-j}\right) t_{n}^{j}\right)+l_{n}(v)= \\
& \frac{a_{m}}{(m-1) !} s_{n, 1} t_{n}^{m-1}+\sum_{j=0}^{m-2} \frac{1}{j !} c_{n, j} t_{n}^{j}+l_{n}(v),
\end{aligned}
$$

where for $n \in \mathrm{N}$ and $0 \leqq j \leqq m-2$,

$$
c_{n, j}=a_{j+1} s_{n, 1}+\sum_{k=j+2}^{m} a_{k}\left(r_{k-j}+\varepsilon_{n, k-j}\right) .
$$

Observe that $s_{n, 1} \rightarrow f\left(X_{1}\right) \neq 0$ and that $c_{n, j}$ remains bounded as $n$ varies. 
Moreover, by (1)

$$
l_{n}(v)=g_{n}(v)=\operatorname{Ad}^{*}\left(\exp t_{n} X\right) g_{n}(v) \rightarrow f(v)=l(v) .
$$

It follows now from (5) and (6) that the sequence $t_{n}, n \in \mathrm{N}$, is bounded. Therefore we can assume $t_{n} \rightarrow t$ for some $t \in \mathrm{R}$. Finally the continuity of

$$
(s, g) \rightarrow \operatorname{Ad}^{*}(\exp s X) g, \mathrm{R} \times \mathrm{g}^{*} \rightarrow \mathrm{g}^{*}
$$

and (1) imply

$$
\begin{aligned}
e\left(\operatorname{Ad}^{*}(\exp (-t X)) f(Y)\right) & \left.=\lim _{n \rightarrow \infty} e\left(\operatorname{Ad}^{*}\left(\exp \left(-t_{n} X\right)\right) \operatorname{Ad}^{*}\left(\exp t_{n} X\right)\right) g_{n}(Y)\right) \\
& =\lim _{n \rightarrow \infty} e\left(g_{n}(Y)\right)
\end{aligned}
$$

for all $Y \in H$. In particular, $e(h(Y))=e\left(\operatorname{Ad}^{*}(\exp (-t X)) f(Y)\right)$ for all $Y \in H$. This proves $\pi_{f} \mid H>\tau$.

LEMMA 3.3. Let $G$ be a non-abelian nilpotent group of the form $\mathrm{R} \ltimes \mathrm{R}^{m}$. Let $\pi \in \hat{G}$ and suppose that for every 1-dimensional subgroup $H$ of $G$ and $\tau \in \hat{H}$, $\pi \prec \operatorname{ind}_{H}^{G} \tau$ implies $\pi \mid H \succ \tau$. Then $G=F \times A$ where $A$ is abelian, $F$ is threadlike and $\pi \mid Z(F)$ is non-trivial.

Proof. The Lie algebra $g$ of $G$ is the form $g=R X \ltimes V$ where $V$ is an $m$-dimensional abelian ideal. Looking at the Jordan decomposition of the nilpotent endomorphism $\operatorname{ad}(X) \mid V$ we see that $V$ decomposes into a direct sum $V=V_{1} \oplus \ldots \oplus V_{r}$ where each $V_{j}$ is an ideal and $\mathrm{g}_{j}=\mathrm{R} X \ltimes V_{j}$ is either abelian (with $\operatorname{dim} V_{j}=1$ ) or threadlike. Of course we can assume that $\operatorname{dim} V_{1} \geqq$ $\operatorname{dim} V_{2} \geqq \ldots \geqq \operatorname{dim} V_{r}$. Then $\operatorname{dim} V_{1} \geqq 2$ as $g$ is non-abelian, and we have to show that $\operatorname{dim} V_{2}=1$.

To this end assume $\operatorname{dim} V_{2} \geqq 2$ and, for $j=1,2$, let $X_{j 1}, X_{j 2} \in \mathfrak{g}_{j}$ such that $\mathrm{R} X_{j 1}$ equals the center of $g_{j}$ and $\left[X, X_{j 2}\right]=X_{j 1}$. Choose $f \in \mathrm{g}^{*}$ with $\pi_{f}=\pi$. Then there exist $\left(a_{1}, a_{2}\right) \neq(0,0)$ such that $f\left(a_{1} X_{11}+a_{2} X_{21}\right)=0$. Setting

$$
Y_{k}=a_{1} X_{1 k}+a_{2} X_{2 k}, k=1,2,
$$

we have $\left[X, Y_{2}\right]=Y_{1}$, so that $Y_{2}$ is central modulo $R Y_{1}$. Now $\pi_{f} \in \widehat{G / R X_{1}}$, and hence $\pi_{f} \mid R Y_{2}$ must be weakly equivalent to some character $\lambda$. Since $H=R Y_{2}$ is non-normal, $1_{G} \prec \operatorname{ind}_{H}^{G} \alpha$ for every $\alpha \in \hat{H}$ (Theorem 2.1). This implies

$$
\pi \prec \pi \otimes \operatorname{ind}_{H}^{G} \alpha=\operatorname{ind}_{H}^{G}(\pi \mid H \otimes \alpha) \sim \operatorname{ind}_{H}^{G}(\lambda \otimes \alpha) .
$$

Therefore, by hypothesis on $\pi, \pi \mid H \sim \hat{H}$. This contradicts $\pi \mid H \sim \lambda$.

We have seen so far that $A=V_{2}+\ldots+V_{r}$ is contained in the center of $G$, and hence $G=F \times A$ where $F=\exp _{1}$. Notice next that $\pi=\rho \times \alpha, \rho \in \hat{F}, \alpha \in \hat{A}$, and 
it is easily verified that $\rho$ satisfies (FPC2) with respect to 1-dimensional subgroups of $F$. By the same argument as above, $\rho \mid \mathrm{R} X_{12} \sim \widehat{\mathrm{R} X_{12}}$, and from this we conclude that $\rho$ cannot be trivial on $\mathrm{R} X_{11}$, the center of $F$.

Lemma 3.1 to 3.3 now combine to give

THEOREM 3.4. Let $G$ be a nilpotent group of the form $G=\mathbf{R} \ltimes \mathbf{R}^{m}$. For $\pi \in \hat{G}$ the following conditions are equivalent:

(i) $\pi$ satisfies (FP2).

(ii) For every 1-dimensional subgroup $H$ of $\mathrm{R}^{m}$ and $\tau \in \hat{H}, \pi \prec \operatorname{ind}_{H}^{G} \tau$ implies $\pi \mid H \succ \tau$.

(iii) $G=F \times A$ where $A$ is abelian, $F$ is threadlike, and $\pi \mid Z(F)$ is non-trivial. In particular, if $G$ is threadlike, then $\pi \in \hat{G}$ has property (FP2) if and only if $\pi \mid Z(G)$ is non-trivial.

Proof. (ii) $\Rightarrow$ (iii) is just Lemma 3.3. To prove (iii) $\Rightarrow$ (i) notice that $\pi=\rho \times \alpha$ for some $\rho \in \hat{F}$ and $\alpha \in \hat{A}$. Let $F=\exp \mathrm{g}_{d}=\mathrm{R} \ltimes \mathrm{R}^{d}$. As $\rho \mid Z(F)$ is non-trivial, there exists $\lambda \in \hat{\mathrm{R}}^{d}$ such that $\rho=\operatorname{ind}_{\mathrm{R}^{d}}^{F} \lambda$, and hence $\pi=\operatorname{ind}_{\mathrm{R}^{d} \times A}^{F}(\lambda \times \alpha)$. Therefore, to prove that $\pi$ has property (FP2), by Lemma 2.7 it suffices to show that for every closed subgroup $H$ of $\mathrm{R}^{d} \times A$ and $\tau \in \hat{H}, \pi \prec \operatorname{ind}_{H}^{G} \tau$ implies $\pi \mid H \succ \tau$. This follows from Lemma 3.2 if $H_{0}$ is not contained in the center of $G$. If $H_{0} \subset Z(G)$, then

$$
\pi \mid H \succ\{\gamma \in \hat{H} ; \gamma|Z(G) \cap H \sim \pi| Z(G) \cap H\}
$$

by Lemma 3.1. But $\pi \prec \operatorname{ind}_{H}^{G} \tau$ yields $\pi\left|H \cap Z(G) \prec\left(\operatorname{ind}_{H}^{G} \tau\right)\right| H \cap Z(G) \sim$ $\tau \mid H \cap Z(G)$, i.e. $\pi|H \cap Z(G) \sim \tau| H \cap Z(G)$.

\section{An Example.}

Let $G$ be a connected and simply connected nilpotent Lie group and $\pi \in \hat{G}$. Comparing the results we obtained in Sections 2 and 3 with those on (FPC1) in [2] leads to the question of whether $\pi$ necessarily has property (FPC1) whenever it satisfies (FPC2). In this final section we present a counterexample.

We consider the 6-dimensional Lie algebra $g$ with Jordan-Hölder basis $X_{1}, \ldots$ $X_{6}$ and non-vanishing Lie brackets

$$
\left[X_{6}, X_{5}\right]=X_{3},\left[X_{6}, X_{4}\right]=X_{2},\left[X_{5}, X_{2}\right]=X_{1}=\left[X_{4}, X_{3}\right]
$$

(in the numbering of $[21], g=g_{6,4}$ ). Let $G=\mathrm{R}^{6}$ with multiplication

$$
\begin{aligned}
& \left(x_{1}, \ldots, x_{6}\right)\left(y_{1}, \ldots, y_{6}\right)= \\
& \left(x_{1}+y_{1}+x_{4} y_{3}+x_{5} y_{2}+x_{4} x_{6} y_{5}+x_{5} x_{6} y_{4}+x_{6} y_{4} y_{5},\right. \\
& \left.x_{2}+y_{2}+x_{6} y_{4}, x_{3}+y_{3}+x_{6} y_{5}, x_{4}+y_{4}, x_{5}+y_{5}, x_{6}+y_{6}\right) .
\end{aligned}
$$


$G$ is a nilpotent group which is isomorphic to the group of upper triangular real $4 \times 4$-matrices and whose Lie algebra is isomorphic to $g$. Let $f_{1}, \ldots, f_{6}$ be the basis of $\mathrm{g}^{*}$ which is dual to $X_{1}, \ldots, X_{6}$. Felix [7,(2.3)] noticed that (FPC1) fails to hold for $\pi_{f_{1}} \in \hat{G}$. We are going to show that nevertheless $\pi_{f_{1}}$ has property (FPC2). In fact, we believe that $\pi_{f_{1}}$ even satisfies (FP2). However, to verify this seems much more tedious. Let

$$
\mathrm{m}=\mathrm{R} X_{6}+\sum_{j=1}^{3} \mathrm{R} X_{j}, \mathfrak{n}=\sum_{j=1}^{3} \mathrm{R} X_{j} \text { and } \mathfrak{z}=\mathrm{R} X_{1},
$$

the center of g. $M=\exp m$ is an abelian normal subgroup of $G$, and $\pi_{f_{1}}$ is induced from a character of $M$. Since the intersection of two connected subgroups of a simply connected nilpotent Lie group is again connected, in order to establish (FPC2) for $\pi_{f_{1}}$ we only have to consider connected subgroups of $M$ (Lemma 2.7). Thus, let $\mathfrak{h}$ be a subalgebra of $m$ and $H=\exp \mathfrak{h}$.

The coadjoint orbits of $G$ in $\mathrm{g}^{*}$ are given in [21]. In particular, for $y=\left(y_{1}, \ldots\right.$, $\left.y_{6}\right) \in G$,

$$
\operatorname{Ad}^{*}(y) f_{1}=f_{1}-y_{5} f_{2}-y_{4} f_{3}+y_{3} f_{4}+y_{2} f_{5}-y_{4} y_{5} f_{6} .
$$

This formula immediately shows that if either $\operatorname{dim} \mathfrak{h}=1$ or $\mathfrak{h} \subset \mathfrak{n}$, then

$$
\operatorname{Ad}^{*}(G) f_{1} \mid \mathfrak{h}=\left\{h \in \mathfrak{h}^{*} ; h\left|\mathfrak{h} \cap \mathfrak{z}=f_{1}\right| \mathfrak{h} \cap \mathfrak{z}\right\} .
$$

On the other hand, if $\pi_{f_{1}} \prec \operatorname{ind}_{H}^{G} \chi_{n}$ denotes the character of $H$ given by $h$, then $h\left|\mathfrak{h} \cap \mathfrak{z}=f_{1}\right| \mathfrak{h} \cap \mathfrak{z}$. Therefore, we can henceforth assume that $\operatorname{dim} \mathfrak{h} \geqq 2$ and $\mathfrak{h} \notin \mathfrak{n}$, i.e. $\mathfrak{h}$ has a basis

$$
X_{6}+A_{1}, A_{2}, \ldots, A_{d}, \text { where } A_{l}=\sum_{j=1}^{3} a_{l j} X_{j} \in \mathfrak{n},
$$

$2 \leqq l \leqq d=\operatorname{dim} \mathfrak{h}$.

Suppose now that $h \in \mathfrak{h}^{*}$ and $\pi_{f_{1}} \prec \operatorname{ind}_{H}^{G} \chi_{h}$. Then there are sequences

$$
g_{n}=\sum_{j=1}^{6} \xi_{n j} f_{j} \in \mathrm{g}^{*} \text { and } x_{n}=\left(x_{n 1}, \ldots, x_{n 6}\right) \in G, n \in \mathrm{N},
$$

such that $g_{n} \mid \mathfrak{h}=h$ and $\operatorname{Ad}^{*}\left(x_{n}\right) g_{n} \rightarrow f_{1}$. The formula for the coadpoint action of $G$ on $\mathrm{g}^{*}$ yields

$$
\begin{gathered}
\xi_{n 1} \rightarrow 1, \\
\xi_{n 2}-x_{n 5} \xi_{n 1} \rightarrow 0, \\
\xi_{n 3}-x_{n 4} \xi_{n 1} \rightarrow 0, \\
\xi_{n 6}+x_{n 5} \xi_{n 3}+x_{n 4} \xi_{n 2}-x_{n 4} x_{n 5} \xi_{n 1} \rightarrow 0,
\end{gathered}
$$


as $n \rightarrow \infty$. Multiplying (4) and (1) and (2) and (3), respectively, and summing up gives

$$
\xi_{n 6} \xi_{n 1}+\xi_{n 2} \xi_{n 3} \rightarrow 0
$$

We have to find $y_{n} \in G$ with

$$
\begin{aligned}
& \operatorname{Ad}^{*}\left(y_{n}\right) f_{1}\left(X_{6}+A_{1}\right)= \\
& -y_{n 4} y_{n 5}+a_{11}-y_{n 5} a_{12}-y_{n 4} a_{13}+y_{n 3} a_{14}+y_{n 2} a_{15} \\
& \rightarrow h\left(X_{6}+A_{1}\right),
\end{aligned}
$$

and for $2 \leqq l \leqq d$,

$$
\operatorname{Ad}^{*}\left(y_{n}\right) f_{1}\left(A_{l}\right)=a_{11}-y_{n 5} a_{12}-y_{n 4} a_{13}+y_{n 3} a_{14}+y_{n 2} a_{15} \rightarrow h\left(A_{l}\right) .
$$

Suppose first that, in addition to (1) - (4) above,

$$
\xi_{n 6}\left(1-\xi_{n 1}\right) \rightarrow 0 \text {. }
$$

Then, choosing $y_{n 5}=-\xi_{n 2}, y_{n 4}=-\xi_{n 3}, y_{n 3}=\xi_{n 4}, y_{n 2}=\xi_{n 5}$ and $y_{n 6}$ and $y_{n 1}$ arbitrary, we obtain from (1), (5) and (8)

$$
\begin{gathered}
\operatorname{Ad}^{*}\left(y_{n}\right) f_{1}\left(X_{6}+A_{1}\right) \\
=\sum_{j=1}^{5} \xi_{n j} a_{1 j}+\xi_{n 6}+a_{11}\left(1-\xi_{n 1}\right)+\xi_{n 6}\left(\xi_{n 1}-1\right)-\left(\xi_{n 6} \xi_{n 1}+\xi_{n 2} \xi_{n 3}\right) \rightarrow
\end{gathered}
$$
$h\left(X_{6}+A_{1}\right)$, since $h\left(X_{6}+A_{1}\right)=g_{n}\left(X_{6}+A_{1}\right)=\sum_{j=1}^{5} \xi_{n j} a_{1 j}+\xi_{n 6}$. Similarly, for $l \geqq 2$,

$$
\operatorname{Ad}^{*}\left(y_{n}\right) f_{1}\left(A_{l}\right)=\sum_{j=1}^{5} \xi_{n j} a_{l j}+a_{l 1}\left(1-\xi_{n 1}\right) \rightarrow h\left(A_{l}\right) .
$$

Notice that (8) holds if either $\xi_{n 6}$ is bounded or $\xi_{n 1}$ is constant, hence in particular if $X_{6} \in \mathfrak{h}$ or $X_{1} \in \mathfrak{h}$. We are now going to verify this.

$\mathfrak{h}$ contains elements $X_{6}+A$ and $B \neq 0$, where

$$
A=\sum_{j=1}^{3} a_{j} X_{j} \text { and } B=\sum_{j=1}^{3} b_{j} X_{j}
$$

If $b_{2}=b_{3}=0$, then $X_{1} \in \mathfrak{h}$. Thus, let $b_{3} \neq 0$, the case $b_{2} \neq 0$ is treated analogously. We can assume $a_{3}=0$. We claim that $b_{2}=0$ or $\xi_{n 2}$ is bounded. Indeed,

$$
\xi_{n 3}=\frac{1}{b_{3}}\left(h(B)-\sum_{j=1}^{2} \xi_{n j} b_{j}\right) \text { and } \xi_{n 6}=h\left(X_{6}+A\right)-\sum_{j=1}^{2} \xi_{n j} a_{j}
$$


and (5) imply

$$
\begin{aligned}
\xi_{n 6} \xi_{n 1} & +\xi_{n 2} \xi_{n 3}=-\frac{b_{2}}{b_{3}} \xi_{n 2}^{2}+\xi_{n 2}\left(\frac{h(B)}{b_{3}}-\left(\frac{b_{1}}{b_{3}}+a_{2}\right) \xi_{n 1}\right) \\
& +h\left(X_{6}+A\right) \xi_{n 1}-a_{1} \xi_{n 1}^{2} \rightarrow 0 .
\end{aligned}
$$

This proves the claim since $\xi_{n 1} \rightarrow 1$. Now, $h(B)=\sum_{j=1}^{3} \xi_{n j} b_{j}$ and $b_{3} \neq 0$. As $b_{2} \xi_{n 2}$ is bounded, we obtain that $\xi_{n 3}$ is bounded. Hence so is $\xi_{n 6}$ in view of (5) and (1).

ACKNOWLEDGEMENT. The author is indebted to the referee for corrections and valuable suggestions.

\section{REFERENCES}

1. B. A. Barnes, The role of minimal idempotents in the representation theory of locally compact groups, Proc. Edinburgh Math. Soc. (2) 23 (1980), 229-238.

2. M. B. Bekka and E. Kaniuth, Topological Frobenius properties for nilpotent groups, Math. Scand. 63 (1988), 282-296.

3. J. Brezin, Geometry and the method of Kirillov, Lecture Notes in Mathematics, Vol. 466 (1975), 13-26.

4. I. D. Brown, Dual topology of a nilpotent Lie group, Ann. Sci. École Norm. Sup. (4) 6 (1973), 407-411.

5. A. L. Carey and W. Moran, Characters of nilpotent groups, Math. Proc. Cambridge Philos. Soc. 96 (1984), 123-137.

6. J. Dixmier, Les $C^{*}$-algèbres et leurs Représentations, Gauthier-Villars, Paris, 1964.

7. R. Felix, Uber Integralzerlegungen von Darstellungen nilpotenter Liegruppen, Manuscripta Math. 9 (1979), 279-290.

8. R. Felix, R.W. Henrichs, and H. L. Skudlarek, Topological Frobenius reciprocity for projective limits of Lie groups, Math. Z. 165 (1978), 19-28.

9. J. M. G. Fell, A Hausdorff topology on the closed subsets of a locally compact non-Hausdorff space, Proc. Amer. Math. Soc. 13 (1962), 472-476.

10. J. M. G. Fell, Weak containment and induced representations of groups. II, Trans. Amer. Math. Soc. 110 (1964), 424-447.

11. F. P. Greenleaf, Amenable actions of locally compact groups, J. Funct. Anal. 4 (1969), 295-315.

12. R. W. Henrichs, Weak Frobenius reciprocity and compactness conditions in topological groups, Pacific J. Math. 82 (1979), 387-407.

13. E. Kaniuth, Ideals in group algebras of finitely generated FC-nilpotent discrete groups, Math. Ann. 248 (1980), 97-108.

14. E. Kaniuth, On topological Frobenius reciprocity for locally compact groups, Arch. Math. 48 (1987), 286-297.

15. E. Kaniuth, On tensor products of representations of motions groups, Arch. Math. 52 (1989), 212-222.

16. A. A. Kirillov, Unitary representations of nilpotent Lie groups, Russian Math. Surveys 17 (1962), 53-104.

17. G. W. Mackey, Induced representations of locally compact groups. I, Ann. Math. (2) 55 (1952), 101-139.

18. C. C. Moore, Groups with finite dimensional irreducible representations, Trans. Amer. Math. Soc. 166 (1972), 401-410.

19. O. A. Nielsen, The failure of the topological Frobenius property for nilpotent Lie groups, Math. Scand. 45 (1979), 305-310. 
20. O. A. Nielsen, The failure of the topological Frobenius property for nilpotent Lie groups. II, Math. Ann. 256 (1981), 561-568.

21. O. A. Nielsen, Unitary Representations and co-adjoint Orbits of Low-dimensional Nilpotent Lie Groups, Queen's Papers in Pure and Appl. Math. 63, Kingston, Ontario, 1983.

22. E. Thoma, Über das reguläre Maß im dualen Raum diskreter Gruppen, Math. Z. 100 (1967), 257-271.

23. J. Tits, Automorphismes à déplacement borné des groupes de Lie, Topology 3 (1964), 97-107.

24. H. Weyl, Úber die Gleichverteillung von Zahlen modulo Eins, Math. Ann. 77 (1916), 313-352.

FACHBEREICH MATHEMATIK/INFORMATIK

UNIVERSITÄT PADERBORN

WARBURGER STR. 100

D-4790 PADERBORN

GERMANY 